\title{
Caracterização de partículas de açaí visando seu potencial uso na construção civil
}

\author{
Characterization of acai waste particles \\ for civil construction use
}

Andrezza de Melo Barbosa ${ }^{1}$, Viviane Siqueira Magalhães Rebelo ${ }^{1}$, Lucieta Guerreiro Martorano $^{2}$,Virginia Mansanares Giacon ${ }^{1}$

\author{
${ }^{1}$ Laboratório de Materiais da Amazônia e Compósitos - LaMAC- PPGEC/FT/UFAM. Av. General Rodrigo Octávio, \\ 6200, Coroado I, CEP: 69080-900, Manaus, Amazonas, Brasil. \\ ${ }^{2}$ Embrapa Amazônia Oriental, Rua Vera Paz, casa 1, Campus UFOPA - Tapajós, Bairro Sale, CEP: 68035-110, Santa- \\ rém, Pará, Brasil. \\ e-mail: andrezzambarbosa.arq@gmail.com, giacon@ufam.edu.br, vivianesiqueira.eng@gmail.com, \\ lucieta.martorano@embrapa.br
}

\begin{abstract}
RESUMO
Na Amazônia, o principal potencial de contribuição para os Objetivos de Desenvolvimento Sustentável (ODS), está no aproveitamento de resíduos da agroindústria para uso na construção civil, como alternativa de novos materiais sustentáveis. Neste contexto, objetivou-se com este trabalho avaliar o potencial uso de partículas de resíduos de açaí (Euterpe oleracea e Euterpe precatoria) caracterizando-as física, química, térmica e morfologicamente. Foram realizados ensaios de densidade por picnometria, verificação de teor de umidade, e dos teores de lignina, celulose e extrativos, de acordo com os parâmetros normativos da TAPPI 204,211 e 222. O resultado da densidade média das partículas é de $1,49 \mathrm{~g} \cdot \mathrm{cm}^{-3}$, com teor de umidade variando de 2 a $6 \%$. Em relação à composição química, observou-se maiores teores de lignina e extrativos, e redução de celulose em relação à outras fibras vegetais. Através da Microscopia Eletrônica de Varredura (MEV), observouse a presença somente do endocarpo do caroço de açaí nas partículas de 8 e 14 Tyler, e maior predominância de fibras nas partículas de 48, 100 e 200 Tyler. O conhecimento das características físico-químicas das partículas de resíduo de açaí faz-se necessário para subsidiar etapas de processamento de compósitos com uso destes materiais. Além disso, a utilização de um resíduo como o caroço do açaí potencializa a redução dos impactos ambientais, fornecendo, principalmente para a construção civil, a possibilidade de produção de novos materiais mais ecológicos, gerando indicadores de sustentabilidade nas edificações da Amazônia.
\end{abstract}

Palavras-chave: açaí, MEV, resíduos agroindustriais, construção sustentável.

\section{ABSTRACT}

In the Amazon, the main potential for contributing to the Sustainable Development Goals (SDG) lies in using agro industry waste in civil construction as an alternative for sustainable materials. In this context, this study aimed to assess the potential use of acai (Euterpe oleracea and Euterpe precatoria) waste particles to create composites that may be used in more sustainable buildings. Density assays were performed using picnometry. The moisture levels were measured and, lignin, cellulose and extractive contents were assessed according to the parameters of TAPPI 204, 211, and 222. Density analyses were performed using picnometry, moisture was analyzed, and lignin, cellulose, and extractive contents were assessed according to the parameters of TAPPI 204, 211, and 222. The results showed that the mean particle density is $1.49 \mathrm{~g} . \mathrm{cm}^{-3}$ and the moisture content ranged from 2 to $6 \%$. Regarding chemical make-up, higher lignin and extractive contents were found, in addition to a reduction in cellulose compared to other plant fibers. Scanning electron microscopy (SEM) revealed the presence of only the endocarp of açaí stone in particles of 8 and 14 Tyler and a higher prevalence of fibers in particles of 48, 100, and 200 Tyler. Knowing the characteristics and behaviors of açaí waste particles is important to lay basis for composite processing stages using this material. Moreover, the use of acai stones reduce the environmental impacts, allowing mainly civil construction to produce new, more ecological materials that lead to sustainability indicators in buildings of the Amazon. 
Keywords: acai, SEM, agro industry waste, sustainable construction.

\section{INTRODUÇÃO}

Em 2015, durante a Assembleia Geral das Nações Unidas, a ONU reuniu os 193 países-membros que se comprometeram a adotar a nova agenda de desenvolvimento sustentável, totalizando 17 objetivos que apontem indicadores de sustentabilidade no planeta Terra, denominados de ODS (Objetivos de Desenvolvimento Sustentável). Dentre esses objetivos, alguns possuem maior aderência às atividades da indústria da construção civil, visando utilizar infraestruturas resilientes, promover a industrialização inclusiva e sustentável e fomentar a inovação, além de tornar as cidades e os assentamentos humanos inclusivos, seguros e sustentáveis [1].

No entanto, o setor da construção civil contribui com cerca de $70 \%$ das emissões de $\mathrm{CO}_{2}$ [2], além da geração de resíduos sólidos urbanos, que englobam os impactos ao meio ambiente, desde a retirada de matéria prima até o descarte de maneira inadequada, em sua fase pós-uso. Como exemplo, podemos citar o valor de $800 \mathrm{~kg} \cdot \mathrm{hab}^{-1}$.ano ${ }^{-1}$ de material descartado em 2014 na cidade São Carlos - SP [3].

Por outro lado, estados como Pará e Amazonas possuem uma alta taxa de desflorestamento de espécies madeireiras que se destinam à construção civil. De acordo com o Instituto de Pesquisas Tecnológicas do Estado de São Paulo - IPT [4] existem 20 espécies mais utilizadas nesse setor, sendo que 100\% delas possuem ocorrência na Amazônia, segundo SOBRAL et al. [5]. Estas são aplicadas principalmente em estruturas de cobertura (50\%), fôrmas para concreto (33\%), forros, pisos e esquadrias (13\%) e casas pré-fabricadas (4\%), representando uma significativa contribuição para ação de desflorestamento.

Neste contexto, a demanda por materiais sustentáveis tem-se intensificado, buscando a inclusão de matérias primas de fontes renováveis, possibilitando a redução do impacto ambiental com menor custo. Assim, materiais de igual ou superior qualidade vêm sendo desenvolvidos, principalmente com o uso de fibras naturais e resíduos agroindustriais para a produção de compósitos [6, 7].

Especificamente na Amazônia, a agroindústria do açaí representa 93\% da produção extrativista vegetal não madeireira do Brasil, sendo o Pará e o estado do Amazonas os dois principais produtores, com produção nacional de $50 \%$ e 33,6\%, respectivamente [8]. De acordo com o IBGE [9], em 2017 foram produzidas cerca de 220 mil toneladas de açaí, e após a remoção da polpa para produção do suco, estima-se que 176 mil toneladas de resíduos foram descartadas, uma vez que $80 \%$ do fruto [10] é composto de fibras e caroço.

Corroborando com as novas necessidades dos chamados materiais eco-friendly e a busca por mitigação dos impactos ambientais oriundos da geração de resíduos do açaí e desflorestamento na Amazônia, objetivou-se caracterizar esse resíduo verificando seu potencial uso na produção de materiais sustentáveis para aplicação na construção civil.

\section{MATERIAIS E MÉTODOS}

\subsection{Materiais}

Foram utilizados caroços de açaí obtidos em feiras livres de Manaus (AM, Brasil) (Figura 1a). Os mesmos foram expostos ao sol durante o período de 8 horas, para retirada natural de água em excesso. Após a présecagem, fez-se uma separação manual, objetivando a retirada de sedimentos e impurezas, tais como talos e folhas.

Em seguida os caroços passaram por um processo de secagem em estufa (Nova Ética, Modelo 404/3D) (Figura 1b), à temperatura de $100{ }^{\circ} \mathrm{C}$, durante 24 horas [11], visando a perda de umidade e do poder germinativo [12]. Após a secagem, um novo peneiramento foi realizado, uma vez que impurezas ainda eram observadas no material. Posteriormente os caroços foram inseridos no moinho de facas tipo Willey, marca Marconi e modelo MA 680 (Figura 1c). Após a trituração, o material particulado foi peneirado utilizando o jogo de peneiras com as granulometrias de 8, 14, 48, 100 e 200 Tyler, ou seja, 2,38, 1,41, 0,37, 0,14 e 0,07 $\mathrm{mm}$, respectivamente (Figura d e e). A separação do material em distintas granulometrias e posterior caracterização ocorreu com intuito de verificar possíveis interferências em suas propriedades e posterior inferência de seu uso em novos materiais na construção civil. 


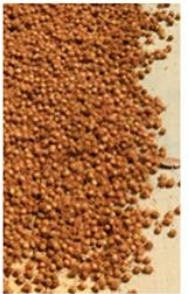

(a)

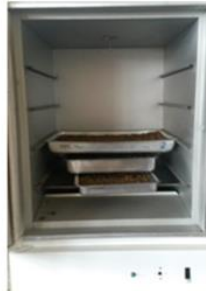

(b)

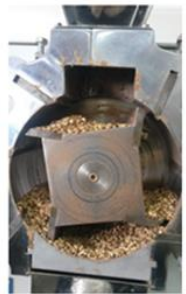

(c)

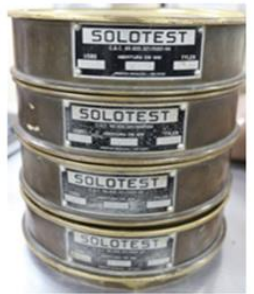

(d)

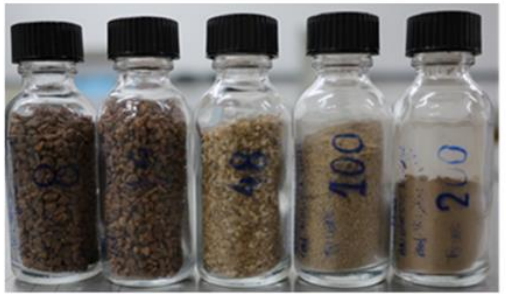

(e)

Figura 1: Preparação do material: a) secagem natural do caroço de açaí; b) secagem em estufa; c) trituração no moinho de facas; d) peneiras usadas no peneiramento; e) separação das partículas por granulometria.

\subsection{Caracterização das partículas dos caroços de açaí}

Para a caracterização das partículas de caroço de açaí, realizaram-se os ensaios de densidade, teor de umidade (\%), ensaios químicos de teor de lignina, celulose e extrativos, além da microscopia eletrônica de varredura (MEV) e análise termogravimétrica (TG e DTG).

As densidades das partículas com diferentes granulometrias (8, 14, 48, 100 e 200 Tyler) foram obtidas utilizando a Picnometria por gás Hélio.

$\mathrm{O}$ teor de umidade presente nos caroços foi obtido utilizando o analisador Halógeno de umidade, MB 35 (Ohaus), com temperatura a $100{ }^{\circ} \mathrm{C}$ e massa mínima de $0,5 \mathrm{~g}$. Para essa análise, optou-se pela separação das partículas em relação à faixa granulométrica considerando 3 composições: 8 e 14 Tyler com ausência de fibras, 48 Tyler com predominância de fibras, bem como uma amostra contendo todas as granulometrias $(8$, $14,48,100$ e 200 Tyler).

Os teores de extrativos e lignina foram obtidos utilizando os documentos normativos TAPPI $204 \mathrm{~cm}$ 97 [13] e TAPPI T 222 om-02[14], respectivamente, e o teor de celulose foi baseada em LEÃO [15].

O teor de Cinzas foi baseado na norma TAPPI T 211[16].

As análises de Microscopia Eletrônica de Varredura (MEV) foram realizadas utilizando-se o equipamento TM 3000 (Hitachi).

A estabilidade térmica das partículas do resíduo de açaí e a sua variação de massa em função da temperatura, foram analisadas pela termogravimetria (TGA) e sua derivada (DTG) no equipamento Shimadzu, modelo SDG 60, com rampa de aquecimento de $10^{\circ} \mathrm{C} \cdot \mathrm{min}^{-1}$, temperatura entre 25 e $1000{ }^{\circ} \mathrm{C}$ em atmosfera inerte com Nitrogênio $[17,18]$. Para tratamento de dados utilizou-se o programa Universal Analisys.

\section{RESULTADOS E DISCUSSÃO}

\subsection{Densidade e teor de umidade}

A densidade das partículas, bem como sua composição, pode influenciar diretamente nas propriedades físicas, químicas e térmicas dos compósitos, além de interferir em respostas mecânicas. Isso ocorre devido à quantidade de vazios existentes nas partículas e de sua área de contato, ou seja, o seu comportamento de adesão e interação com a matriz [19]. Na tabela 1, observam-se os valores de densidade das partículas de resíduo de açaí, nas diferentes granulometrias estudadas. 
Tabela 1: Densidade das partículas de resíduo de açaí.

\begin{tabular}{c|c}
\hline GRANULOMETRIA & DENSIDADE \\
\hline Tyler & g.cm $^{-3}$ \\
\hline 8 & 1,496 \\
\hline 14 & 1,481 \\
\hline 48 & 1,477 \\
\hline 100 & 1,527 \\
\hline 200 & 1,514 \\
\hline
\end{tabular}

Os valores da densidade encontrados nas amostras são semelhantes às densidades de fibras vegetais como juta e linho [20], e que são amplamente utilizadas em materiais compósitos.

Em relação ao teor de umidade, observou-se que menores teores ocorreram em granulometrias de 8 e 14 Tyler, e o maior teor ocorreu na granulometria de 48 Tyler (Tabela 2). Esse resultado pode estar relacionado com a presença predominante de fibras nessa granulometria, conforme observado na figura 2 , uma vez que estas são mais higroscópicas, aumentado a absorção de água do material.

Tabela 2: Teor de umidade das partículas

\begin{tabular}{c|c|c|c|c}
\hline GRANULOMETRIA & $\begin{array}{c}\text { MASSA } \\
\text { INICIAL }\end{array}$ & $\begin{array}{c}\text { MASSA } \\
\text { FINAL }\end{array}$ & $\begin{array}{c}\text { PERDA DE } \\
\text { MASSA }\end{array}$ & TEOR DE UMIDADE \\
\hline (Tyler) & $(\mathbf{g})$ & $(\mathbf{g})$ & $\mathbf{( g )}$ & $\mathbf{( \% )}$ \\
\hline 8 e 14 & 3,51 & 3,41 & 0,10 & 2,78 \\
\hline $8,14,48,100,200$ & 3,51 & 3,39 & 0,12 & 3,42 \\
\hline 48 & 3,52 & 3,31 & 0,20 & 5,76 \\
\hline
\end{tabular}

É importante salientar que o percentual ideal de teor de umidade para aplicação dos materiais lignocelulósicos em compósitos, de acordo com MALONEY [21], é em torno de 5\%. O uso de materiais lignocelulósicos com alto teor de umidade podem implicar em perdas de propriedades mecânicas nos compósitos devido à formação de bolhas que ocorrem durante o seu processamento [22, 23]. Por outro lado, baixos valores de teor de umidade podem diminuir o transporte de calor para a região central do compósito, gerando um material heterogêneo, e, portanto, com perdas de propriedades mecânicas [24, 25].

\subsection{Caracterização Química: Teor de lignina, celulose e extrativos}

Na tabela 3 são apresentadas as composições químicas dos resíduos de açaí (caroço e fibra), apenas fibras de açaí, fibras de madeira das espécies Cumaru [26], entre outras.

Tabela 3: Composição química dos resíduos de açaí (caroço + fibra) comparada à outras fibras naturais.

\begin{tabular}{l|c|c|c|c|c}
\hline \multicolumn{1}{c|}{ MATERIAL } & EXTRATIVOS (\%) & CELULOSE (\%) & CINZAS (\%) & LIGNINA (\%) & REFERÊNCIA \\
\hline $\begin{array}{l}\text { Resíduo de Açaí } \\
\text { (caroço + fibra) }\end{array}$ & $16,64 \pm 0,73$ & $36,13 \pm 4,32$ & $1,57 \pm 0,02$ & $47,92 \pm 4,41$ & $\begin{array}{c}\text { Presente } \\
\text { trabalho }\end{array}$ \\
\hline $\begin{array}{l}\text { Fibra de Açaí } \\
\text { E. } \text { oleracea Mart. }\end{array}$ & - & 46,51 & - & 30,35 & {$[27]$} \\
\hline $\begin{array}{l}\text { Fibra de Açaí } \\
\text { E. precatoria Mart. }\end{array}$ & - & 73,92 & - & 10,16 & {$[28]$} \\
\hline $\begin{array}{l}\text { Fibra de Madeira } \\
\text { Cumaru }\end{array}$ & 18,32 & 46,79 & 1,72 & 34,29 & {$[29]$} \\
\hline Fibra de coco & 2,33 & 45,47 & 1,34 & 31,83 & {$[30]$} \\
\hline Fibra de Sisal & 9,27 & 59,18 & 1,95 & 6,97 & {$[30]$} \\
\hline Eucalipto & 15,88 & 40,64 & 1,35 & 17,12 & {$[31]$} \\
\hline
\end{tabular}


O resíduo de açaí estudado apresentou o maior teor de lignina em relação aos demais materiais. Esse é um fator importante, uma vez que a lignina possui uma estrutura amorfa polimérica [28, 32] que contribui não só para a proteção a ataques químicos, mas também pode proporcionar um maior tempo de degradação para o material. Esse resultado pode indicar melhor resposta de durabilidade em compósitos constituídos por caroço e fibras, quando comparados à compósitos produzidos apenas com fibras de açaí.

Quanto ao percentual de celulose, cuja principal característica é conferir maior rigidez e resistência à tração [33], observou-se menor quantidade no resíduo de açaí quando comparado somente à fibra. Assim, o uso de caroços em materiais compósitos pode implicar em propriedades mecânicas inferiores quando comparados aos confeccionados somente com as fibras do fruto, a exemplo dos estudos de QUIRINO [28] e MESQUITA [27].

Em relação ao maior teor de extrativos presente no resíduo de açaí, quando comparado às fibras de coco, sisal ou madeira Cumaru, este poderá dificultar a adesão entre as partículas do resíduo de açaí e resinas termofíxas, na produção de compósitos poliméricos. Segundo LIMA et al. [34], isso se dá pelo fato dos extrativos interferirem na reação de polimerização da resina, uma vez que estes são compostos em geral por graxas e óleo, diminuindo a interação entre matriz e reforço [35].

\subsection{Caracterização Morfológica: Microscopia Eletrônica de Varredura (MEV)}

Nas figuras 2a (1 e 2) e 2b (1 e 2), referentes às granulometrias 8 e 14 Tyler, respectivamente, não são observadas fibras. Por outro lado, essas fibras estão presentes em amostras a partir de 48 Tyler, conforme as figuras 2 c (1 e 2), 2 d (1 e 2) e 2e (1e 2). Essas microscopias confirmam a influência de presença de fibra no aumento do teor de umidade conforme discutido no item 3.1 . 


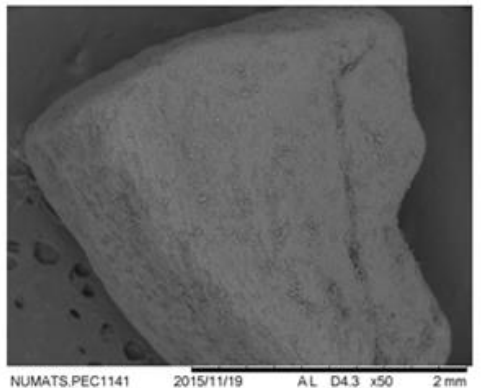

(a.1)

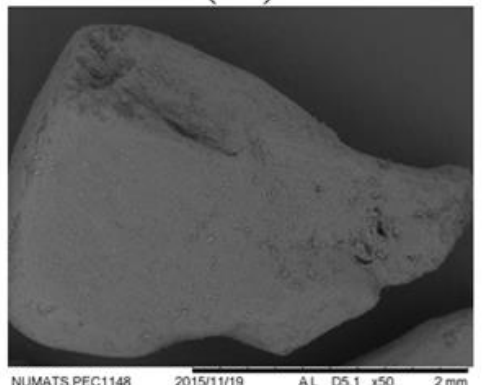

(b.1)

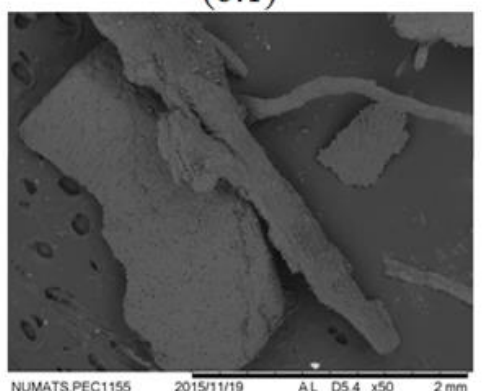

(c.1)

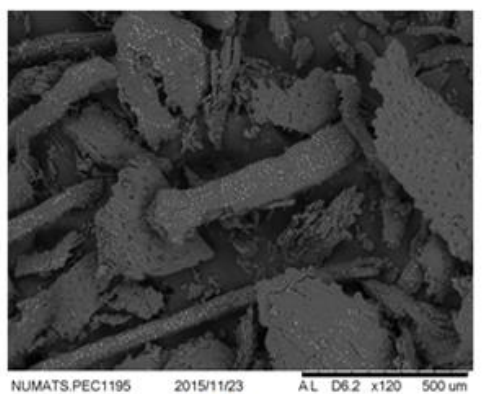

(d.1)

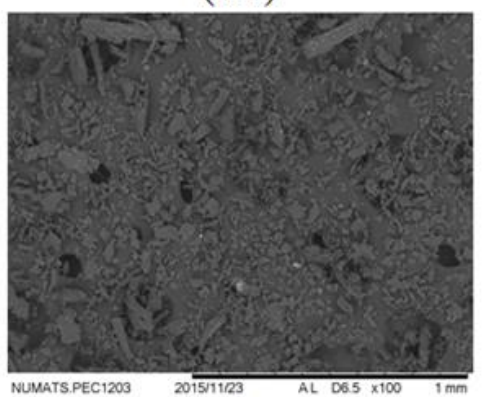

(e.1)

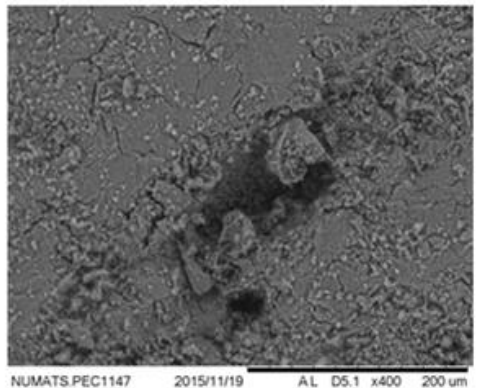

(a.2)

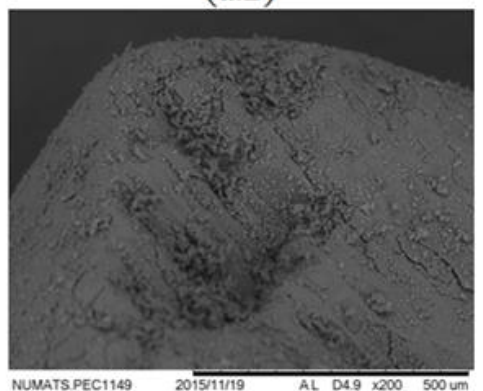

(b.2)

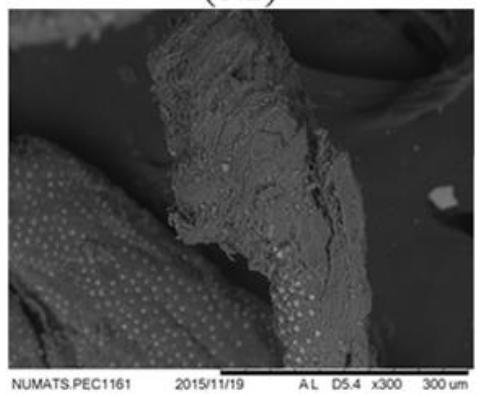

(c.2)

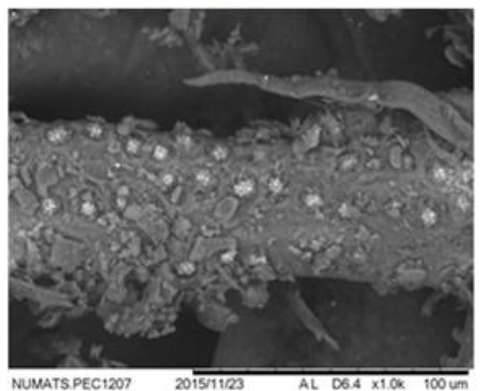

(d.2)

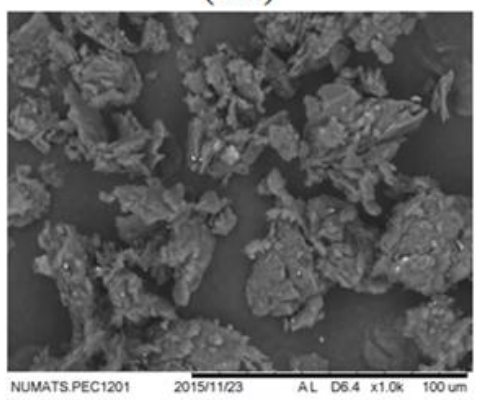

(e.2)

Figura 2: Micrografias de MEV das partículas de resíduo de açaí, com diferentes granulometrias: a.1) 8 Tyler com aumento de 50x; a.2) 8 Tyler com aumento de 400x; b.1) 14 Tyler com aumento de 50x; b.2) 14 Tyler com aumento de 200x; c.1) 48 Tyler com aumento de 50x; c.2) 48 Tyler com aumento de 300x; d.1) 100 Tyler com aumento de 120x; d.2) 100 Tyler com aumento de 1000 x; e.1) 200 Tyler com aumento de 100x; e.2) 200 Tyler com aumento de 1000x. 
Para a partícula de 8 Tyler, cuja morfologia é semelhante à de 14 Tyler, observa-se uma superfície irregular, que de acordo com COSTA et al. [36], está associada à deformação sofrida durante o corte e trituração no moinho de facas, bem como devido ao atrito entre partículas. Na Figura 2d2, observa-se que a fibra apresenta protrusões ricas em Sílica $\left(\mathrm{SiO}_{2}\right)$, de acordo com LIMA et al. [37], e podem ser melhor visualizadas na Figura 3.

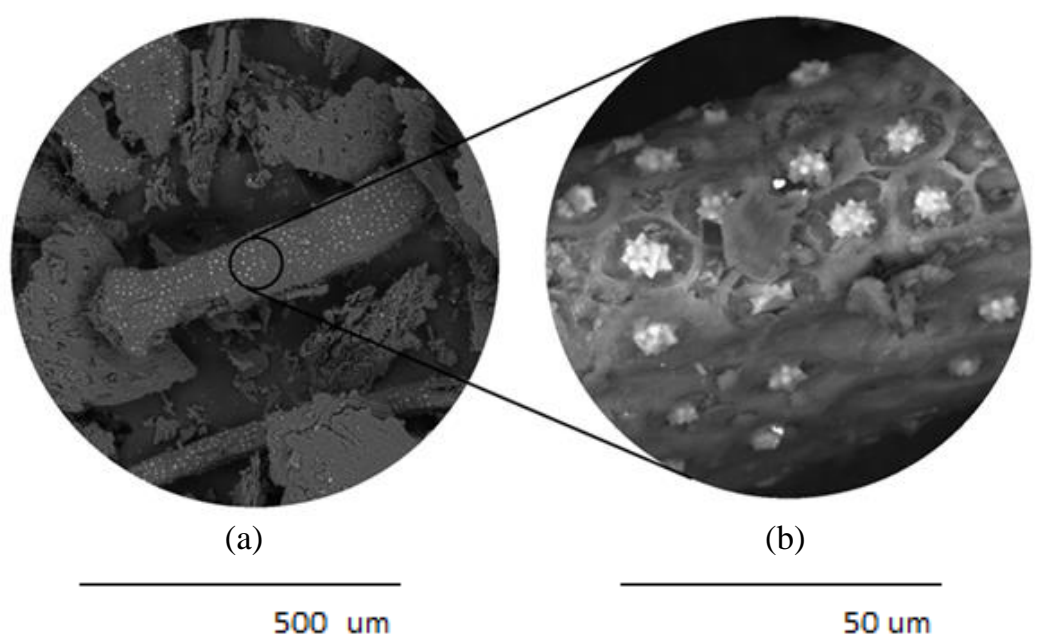

Figura 3: Micrografia de MEV da superfície das fibras: a) fibras; b) protrusões [38]

De acordo com MESQUITA et al. [39] a presença de sílica proporciona à fibra maior rigidez e benefícios às suas características mecânicas. Por outro lado, estudos realizados por MIRANDA et al. [40] exemplificam a aplicação de tratamentos químicos em fibras para retirada da sílica, aumentando sua rugosidade, desde que usado na intensidade adequada para não causar desfibrilação e perda de resistência. Esses tratamentos poderiam contribuir para melhorias nas características mecânicas de compósitos em virtude de fatores como melhor interação entre os componentes.

\subsection{Caracterização Térmica: TGA e DTG}

Na Figura 4 estão representados os resultados do ensaio de degradação térmica de amostras in natura. Para as maiores partículas de 8 e 14 Tyler, contendo apenas o endocarpo, o perfil de degradação por temperatura foi semelhante. Para essas granulometrias, o evento relacionado à perda de água não é observado [41]. Por outro lado, esse evento é observado nas amostras de 48, 100 e 200 Tyler, provavelmente devido à presença de fibras nessas granulometrias, uma vez que estas são mais hidrofílicas [42]. 


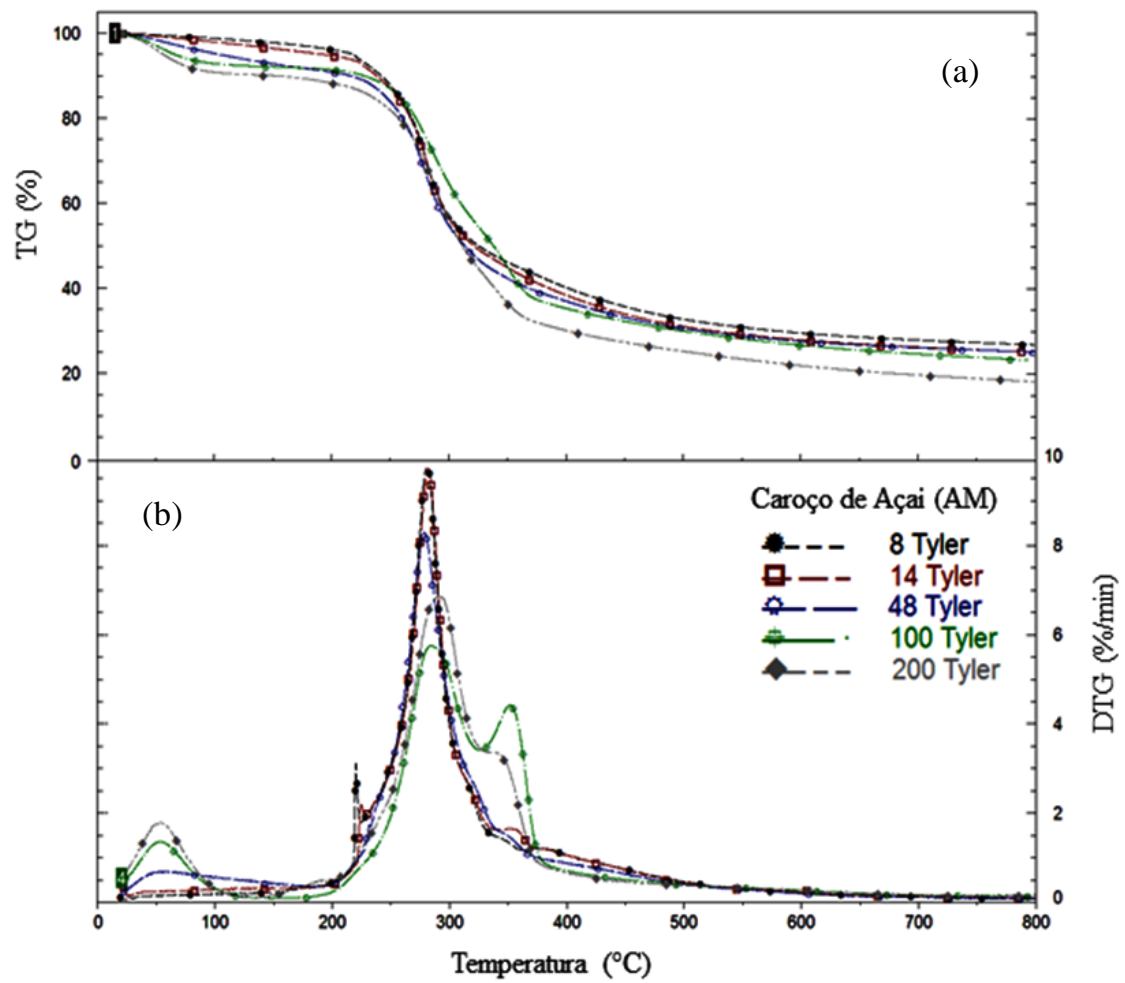

Figura 4: Curvas de TG (a) e DTG (b) de todas as partículas estudadas do caroço de Açaí.

A segunda etapa da degradação ocorre no intervalo de 195 e $230{ }^{\circ} \mathrm{C}$, para todas as granulometrias, com pico de temperatura em $220^{\circ} \mathrm{C}$, e está relacionado à degradação da hemicelulose [41].

O segundo pico com maior intensidade de variação de massa se deu até $360{ }^{\circ} \mathrm{C}$, totalizando uma redução de $51 \%$ para as partículas de 8 Tyler, e $47 \%$ para as de 14 Tyler. Este pico está relacionado à decomposição da celulose [41] com temperaturas máximas de $280{ }^{\circ} \mathrm{C}$, para este intervalo.

Já a terceira e última etapa ocorreu por volta dos $330{ }^{\circ} \mathrm{C}$, com o terceiro pico de degradação aos $383{ }^{\circ} \mathrm{C}$ para 8 Tyler e aos $350{ }^{\circ} \mathrm{C}$ para 14 Tyler, com perdas de massa de 5 e $6 \%$ atribuído à decomposição dos resíduos tais como gorduras do endocarpo [41, 42].

Nas curvas TG/DTG das partículas de 48 Tyler observa-se um primeiro evento até aproximadamente $190^{\circ} \mathrm{C}$, com perda de massa de $9 \%$, referente à perda de umidade, reforçando a inerência hidrofílica das fibras, presentes em amostras dessa granulometria. A partir de $200{ }^{\circ} \mathrm{C}$ há uma intensa perda de massa de cerca de $47 \%$ com pico de $278{ }^{\circ} \mathrm{C}$. Um pico de baixa intensidade ocorre posteriormente, até $365^{\circ} \mathrm{C}$, com uma variação de $5 \%$.

As amostras de granulometrias de 100 e 200 Tyler, apresentaram perfis semelhantes e também três etapas de perda de massa. Até $100{ }^{\circ} \mathrm{C}$ houve a diminuição de massa por perda de água com pico de decomposição aos $50{ }^{\circ} \mathrm{C}$ e redução de 7 a $9 \%$. Posteriormente ocorreu um evento de maior intensidade, iniciando em $200{ }^{\circ} \mathrm{C}$ com picos de $280{ }^{\circ} \mathrm{C}$ e $290{ }^{\circ} \mathrm{C}$ e perda de 36 e $43 \%$ para 100 e 200 Tyler, respectivamente, relacionado à degradação da hemicelulose. Já o terceiro evento, entre 325 e $380{ }^{\circ} \mathrm{C}$, com perda de massa de $19 \%$ para granulometria de 100 Tyler e $14 \%$ para 200 Tyler, e temperaturas de pico de $350{ }^{\circ} \mathrm{C}$ e $340{ }^{\circ} \mathrm{C}$, estão relacionados à degradação de celulose.

A partir desses resultados verifica-se que amostra com presença ou não de fibras apresentam estabilidade térmica até aproximadamente $200^{\circ} \mathrm{C}$.

\section{CONCLUSÕES}

As características observadas em amostras de resíduos de açaí, como teor de umidade, densidade e temperatura de degradação indicam seu potencial para a produção de materiais compósitos aplicados na construção civil.

Os resultados de porcentagem de extrativos indicam uma necessidade de tratamento químico visando 
um aumento da adesão das partículas em compósitos termofixos.

O uso das partículas do resíduo de açaí englobando tanto o caroço quanto as fibras potencializa a mitigação dos impactos ambientais gerados pela agroindústria e das forçantes de desflorestamento da floresta Amazônica, substituindo uma parte da cadeia de consumo de madeira, por ações de reaproveitamento de materiais que geram indicadores de sustentabilidade nas edificações

\section{AGRADECIMENTOS}

Os autores expressam seus agradecimentos à CAPES, pelo apoio concedido à pesquisa, e à COPPE/UFRJ pelo apoio à realização dos ensaios de picnometria, Termogravimetria e Microscopia Eletrônica de Varredura.

\section{BIBLIOGRAFIA}

[1] ONU, Organização das nações unidas (2015), Assembleia Geral das Nações Unidas, Transformando Nosso Mundo: A Agenda 2030 para o Desenvolvimento Sustentável (ODS), Rio de Janeiro.

[2] UN-HABITAT (2016), World cities report 2016: Urbanization and development, Emerging futures, Kenya.

[3] CBCS, Conselho brasileiro de construção sustentável (2014), Aspectos da construção sustentável no Brasil e promoção de políticas públicas, São Paulo.

[4] IPT, Instituto de Pesquisas Tecnológicas do Estado de São Paulo (2013), Catálogo de madeiras brasileiras para a construção civil, São Paulo.

[5] SOBRAL, L., VERÍSSIMO, A., LIMA, E., et al., Acertando o alvo 2: consumo de madeira amazônica e certificação florestal no Estado de São Paulo, Belém, Imaflora, 2002.

[6] REDE NOSSA SÃO PAULO, REDE SOCIAL BRASILEIRA POR CIDADES JUSTAS E SUSTENTÁVEIS, INSTITUTO ETHOS DE EMPRESAS E RESPONSABILIDADE SOCIAL (2012), Programa cidades sustentáveis. 5 ed., São Paulo.

[7] LAHR, F. A. R., SAVASTANO JUNIOR, H., FIORELLI, J. (Org.), "Use of amazon vegetable fibers' waste and woods for the production of polymeric composites", In: Non-conventional Building Materials based on agro-industrial wastes, São Paulo, pp. 313-328, 2015.

[8] IBGE, Instituto Brasileiro de Geografia e Estatística (2014), Produção da extração vegetal e silvicultura, v. 29, Rio de Janeiro.

[9] IBGE, Instituto Brasileiro de Geografia e Estatística (2017), Produção da extração vegetal e silvicultura PEVS, Rio de Janeiro.

[10] BARREIRA, R. M, Caracterização físico-química do endocarpo do açaí (Euterpe oleracea mart.) para aplicação em sintese de poliuretana, Dissertação de M.Sc., Universidade Federal do Pará, Belém, PA, Brasil, 2009.

[11] LEÃO, N. V. M., OHASHI, S. T., FREITAS, A. D. D., et al., (2011), Colheita de Sementes e Produção de Mudas de Espécies Florestais Nativas, Documentos, 374, Belém.

[12] MORAES, J. R. S. C., OLIVEIRA, C. M., FERREIRA, E. M., et al., "Análise do poder germinativo de sementes de Açaí (Euterpe oleracea MART.), em diferentes tipos de tratamentos, utilizando serragem como substrato”, In: 64 ${ }^{a}$ Reunião Anual da SBPC, São Luiz, Maranhão, Brasil, 22-27 Julho 2011.

[13] TAPPI (1997), T 204 cm-97: Solvent extractives of wood and pulp, Technical Association of the Pulp and Paper Industry.

[14] TAPPI (2002), T222 om-02: Acid-insolub lignin in wood and pulp, Technical Association of the Pulp and Paper Industry.

[15] LEÃO, M. A., Fibras de Licuri: Um reforço alternativo de compósitos poliméricos, Dissertação, Universidade Federal do Rio Grande do Norte, Natal, RN, Brasil, 2008.

[16] TAPPI (2002), T 211 om-02: Ash in wood, pulp, paper and paperboard: combustion at $525{ }^{\circ} \mathrm{C}$, Technical Association of the Pulp and Paper Industry.

[17] CANEVAROLO JÚNIOR, S. V, Ciência dos polímeros: um texto básico para tecnólogos e engenheiros, 2 ed., São Paulo, Artliber Editora, 2006.

[18] MOTHÉ, C. G., AZEVEDO, A. D., Análise térmica de materiais, São Paulo, Editora Artliber, 2009. 
[19] ELEOTÉRIO, J. R., Propriedades físicas e mecânicas de painéis MDF de diferentes densidades e teores de resina, Dissertação de M.Sc., Escola Superior de Agricultura “Luiz Queiroz", USP, Piracicaba, SP, Brasil, 2000.

[20] PEREIRA, P. H. F., ROSA, M. F., CIOFF, M. O. H., et al., "Vegetal fbers in polymeric composites: a review”, Polímeros, São Carlos, v. 25, n.1, pp. 9-22, 2015.

[21] MALONEY, T. M. Modern particleboard \& Dry-process fiberboard manufacturing. 3. Ed. San Francisco: Miller Freeman, 1989. 672p.

[22] KELLY, M. W., Critical literature review of relationships between processing parameters and physical properties of particleboard, Madison, WI: USDA, Forest Service, Forest Products Laboratory, 1977.

[23] HILLIG, E., Qualidade de chapas aglomeradas estruturais, fabricadas com madeiras de Pinus, Eucalipto e Acácia negra, puras ou misturadas, coladas com tanino-formaldeído, Dissertação de M.Sc., Universidade Federal de Santa Maria, Santa Maria, RS, Brasil, 2000.

[24] MALONEY, T. M., Modern particleboard \& dry-process fiberboard manufacturing, San Francisco, Miller Freeman, 1989.

[25] HASELEIN, C. R., CALEGARI, L., BARROS, M. V., et al., "Resistência mecânica e à umidade de painéis aglomerados com partículas de madeira de diferentes dimensões", Ciência Florestal, Santa Maria, v. 12, n.2, pp. 127-134, Jul. 2002.

[26] ZAU, M. D. L., VASCONCELOS, R. P., GIACON, V. M., et al., “Avaliação das Propriedades Química, Física e Mecânica de Painéis Aglomerados Produzidos com Resíduo de Madeira da Amazônia - Cumaru (Dipteryx Odorata) e Resina Poliuretana à Base de Óleo de Mamona”, Polímeros, São Carlos, v. 24, n. 6, pp.726-732, 2014.

[27] MESQUITA, A. de L., Estudos de processos de extração e caracterização de fibras do fruto do Açaí (Euterpe oleracea MART.) da Amazônia para produção de ecopainel de partículas homogêneas de média densidade, 2013, 166 f., Tese de D.Sc, Universidade Federal do Pará, UFPA, Belém, PA, Brasil, 2013.

[28] QUIRINO, M. G. Q., Estudo de matriz polimérica produzida com resina natural e fibra da semente de açaí (Euterpe precatória), Dissertação de M.Sc., Universidade Federal do Amazonas, UFAM, Manaus, AM, Brasil, 2010.

[29] LIMA, M. D. F., Utilização de resíduos da espécie Dipteryx polyphylla (Cumarurana), Dipteryx odorata (Cumaru) e Brosimum parinarioides (Amapá) na produção de painéis de madeira aglomerada com resina poliuretana à base de óleo da mamona, Dissertação de M.Sc., Universidade Federal do Amazonas, UFAM, Manaus, AM, Brasil, 2012.

[30] SALAZAR, V. L. P., LEÃO, A. L., “Aproveitamento da fibra de coco com látex para aplicação em assentos automobilísticos", In: Congresso interamericano de engenharia sanitária e ambiental, Porto Alegre, Brasil, 2000.

[31] TEIXEIRA, C. M., MARTINS, M. P., YAMAMOTO, H., et al., "Caracterização Química de Resíduos de Eucalyptus sp. de Floresta de Curta Rotação para a Produção de Bioenergia”, Revista virtual de química, v.8, n. 4, pp. 1693-1701, Set., 2016.

[32] DOREZ, G., FERRY, L., SONNIER R., et al., "Effect of cellulose, hemicellulose and lignin contents on pyrolysis and combustion of natural fibers", Journal of Analytical and Applied Pyrolysis, v. 107, pp. 323-331, Mai. 2014.

[33] YAN, L., KASAL, B., HUANG, L., “A review of recent research on the use of cellulosic fibres, their fibre fabric reinforced cementitious, geo-polymer and polymer composites in civil engineering", Composites Part B: Engineering, v. 92, n. 1, pp. 94-132, Mai. 2016.

[34] LIMA, C. K. P., MORI, F. A., MENDES, L. M., et al., "Características anatômicas e química da madeira de clones de Eucalyptus", Cerne, Lavras, v. 13, n. 2, pp. 123-129, Set. 2007.

[35] ALBINO, V. C. S., MORI, F. A., MENDES, L. M., "Influência das características anatômicas e do teor de extrativos totais da madeira de Eucalyptus grandis W. Hill ex Maiden na qualidade da colagem", Ciência Florestal, Santa Maria, v. 22, n. 4, pp. 803-811, Out. 2012.

[36] COSTA, R. G., ANDREOLA, K., MATTIETTO, R. A., et al., "Effect of operating conditions on the yield and quality of açaí (Euterpe oleracea Mart.) powder produced in spouted bed", LWT - Food Science and Technology, v.64, pp.1196-1203, Dez. 2015. 
[37] LIMA, A. M. de, CHRISTOFORO, A. L., FARIA, L. J. G., et al., "Influence of alkaline mercerization of treatment in the tensile strength of Açaí fiber", In: Non-conventional Building Materials based on agroindustrial wastes, São Paulo, pp. 125-142, 2015.

[38] NASCIMENTO, D. C. O., FERREIRA, A., MONTEIRO, S. N., et al., "Studies on the characterization of piassava fibers and their epoxy composites", Composites, n. 43, pp. 353-362, Dez. 2011.

[39] MIRANDA, C. S., FIUZA, R. P., CARVALHO, R. F., et al., "Efeito dos tratamentos superficiais nas propriedades do bagaço da fibra de piaçava (Attalea funifera Martius)", Química Nova, v. 38, n. 2, pp. 161165, Nov. 2015.

[40] RAMBO, M. K. D., RAMBO, M. C. D., RODRIGUES, K., et al., "Study of thermo-gravimetric analysis of different lignocellulosic biomass using principal component analysis", Ciência e Natura, v. 37, pp. 862868, Set. 2015.

[41] MARTINS, M. A., MATTOSO, L. H. C., PESSOA, J. D. C., "Comportamento térmico e caracterização morfológica das fibras de mesocarpo e caroço do açaí (Euterpe oleracea Mart.)", Revista Brasileira de Fruticultura (Impresso), v. 31, pp. 1150-1157, Dez. 2009.

[42] POLETTO, M., ORNAGHI JÚNIOR, H. L., ZATTERA, A. J., "Native cellulose: structure, characterization and thermal properties”, Materials, n. 7, pp. 6105-6119, Ago. 2014.

\section{ORCID}

Andrezza de Melo Barbosa

https://orcid.org/0000-0002-9403-3238

Vivivane Siqueira Magalhães Rebelo

https://orcid.org/0000-0002-1505-0727

Lucieta Guerreiro Martorano

https://orcid.org/0000-0003-3893-3781

Virginia Mansanares Giacon

https://orcid.org/0000-0003-3628-7317 